\title{
EXCELÊNCIA OPERACIONAL NO PROCESSO PRODUTIVO DE UMA MINERAÇÃO *
}

\author{
Luciano Almeida de Freitas ${ }^{1}$ \\ Luciano José Vieira Franco ${ }^{2}$ \\ Jussara Fernandes Leite ${ }^{2}$ \\ Fernanda Maciel Zeferino ${ }^{2}$ \\ Fernando Marinho ${ }^{2}$ \\ Nilo Antunes Ferreira ${ }^{2}$ \\ Márcio Roberto Rodrigues ${ }^{2}$
}

\section{Resumo}

Este artigo tem como objetivo verificar os resultados da aplicação de ações que almejam a excelência no processo de carga, descarga e transporte de minério ferro de uma empresa situada na Região do Médio Piracicaba em Minas Gerais. Esta pesquisa é um estudo de caso de natureza exploratória e documental. No desenvolvimento deste estudo foram analisados os documentos da empresa, que referem-se às atas de reunião e relatórios gerenciais. As reuniões foram realizadas pelos gestores e supervisores do processo de carga, descarga e transporte de minério para identificar falhas e apresentar ações que almejassem a excelência no processo produtivo. Por fim, os resultados foram verificados e identificou um aumento no índice da utilização física dos caminhões de $11,46 \%$, aumento da produtividade horária de $27,5 \%$ e um crescimento da movimentação total de produção dos caminhões de $23,92 \%$. Com isso, pode-se verificar os ganhos com a aplicação das ações implementadas, que almejam a excelência nas operações.

Palavras-chave: Excelência. Processo. Desempenho. Performance.

\section{OPERATIONAL EXCELLENCE IN THE PRODUCTION PROCESS OF A MINING Abstract}

This article has the objective to verify the results of the application of actions that aim at excellence in the process of charging, recharging and transporting iron ore from a company located in the Médio Piracicaba Region of Minas Gerais. This research is a case study of an exploratory and documentary nature. In the development of this study we analyzed the company's documents, which refer to meeting minutes and management reports. The meetings were carried out by the managers and supervisors of the process of charging, recharging and transportation of ore to identify failures and present actions that aim at excellence in the production process. Finally, the results were verified and identified an increase in the physical utilization rate of the trucks of $11.46 \%$, an increase in hourly productivity of $27.5 \%$ and a growth of the total truck production movement of $23.92 \%$. With this, one can verify the gains with the application of the implemented actions, that aim at the excellence in the operations.

Keywords: Excellence. Process. Acting. Performance.

1 Graduando em Engenharia de Produção, Fundação Presidente Antônio Carlos, de Barão de Cocais, Minas Gerais, Brasil.

2 Engenheiro, Professor da Fundação Presidente Antônio Carlos, Conselheiro Lafaiete, Minas Gerais, Brasil. 


\section{INTRODUÇÃO}

As empresas do setor de minério de ferro no Brasil passam por um momento de crise, uma vez que seu produto foi atingido pela queda da demanda pelo seu maior cliente, a China. Esse fato afetou diretamente o preço do produto, que segundo informações do Ministério do Desenvolvimento, Indústria e Comércio (MDIC) citado por Francia ${ }^{1}$, a China comprou 47,3\% a menos de minério em 2015 se comparado com o mesmo período em 2014. Com a diminuição da demanda, houve uma crescente oferta do produto no cenário mundial, fazendo com que o preço da tonelada do minério começasse a despencar de US $\$ 140,00 /$ ton. em janeiro de 2014 , para pouco mais de US $\$ 50,00 /$ ton. em janeiro de 2016 . Nesse cenário, as empresas do setor de mineração, especificamente de minério de ferro, buscam ser mais competitivas no mercado. Assim, diferenciar-se da concorrência em termos de qualidade, serviço, flexibilidade, inovação e respostas rápidas às alterações do mercado, tornou-se uma das características mais importante da gestão estratégica das organizações. Desta forma, é fundamental a criação de formas para medir e avaliar o desempenho dos processos, isso para identificar desvios e poder saná-los a fim de obter resultados eficazes, ou seja, a excelência da organização.

A busca da excelência, segundo Montenegro², não deve ser um esforço momentâneo nas organizações. Trata-se de um processo dinâmico e contínuo de mudança, em que cada um deve fazer a sua parte, contribuindo para que a empresa se torne cada vez melhor e obtenha o almejado reconhecimento como organização excelente.

Montenegro ${ }^{2}$ ressalta que ter um objetivo firme, constante e relacionado à excelência é o primeiro passo de uma jornada que deve se traduzir em expectativa, metas e planos. É preciso, sobretudo, método para transformar o objetivo em realidade. Uma meta que não seja sustentada por um método leva frequentemente, ao desestímulo e à frustração. Em linhas gerais, a busca pela excelência é um desafio de como ser mais competitivo, como utilizar a capacidade produtiva e buscar ganhos reais de desempenho. Logo, a excelência diz respeito a gerar valor de forma excepcional e sustentável, para todos os envolvidos no negócio.

Detalhadamente, Montenegro ${ }^{2}$ explica que a excelência, por definição, sugere algo de primeira classe, algo superior excepcional. Ser excelente é se destacar, ir além, superar expectativas. Excelência representa também um esforço contínuo, em que cada momento é uma oportunidade única, de superação, de revelar atitudes que conduzam aos melhores resultados. É um processo cíclico e constante de estabelecer um desafio para nós mesmos, de ser sempre melhor. Cada conquista representa um ponto de partida para outra. Esse lado da excelência diz respeito às motivações e ao comportamento.

A Fundação Nacional da Qualidade - $\mathrm{FNQ}^{3}$ esclarece que as organizações excelentes são aquelas que adotam as melhores práticas de gestão e conseguem, com isso, os melhores resultados, fazendo delas, referência para outras organizações.

Sobre excelência, Corrêa ${ }^{4}$ afirma que muitas vezes refere-se a um resultado excelente como padrão classe mundial, em que os resultados alcançados são comparáveis aos melhores resultados obtidos no mundo. Isso pressupõe, portanto, que a organização acompanha o desempenho dos concorrentes em nível mundial. Ser competitivo é ser melhor que a concorrência nos aspectos valorizados pelo cliente.

$\mathrm{Na}$ caminhada em busca da excelência, Juran ${ }^{5}$ exprime que a empresa se depara frequentemente com a necessidade de empreender mudanças. Se as coisas são feitas da mesma forma, não se pode esperar evolução significativa dos resultados. Mudanças substanciais de desempenho exigem mudanças igualmente radicais de prática.

Por meio de mudanças, as melhorias são realizadas nos processos. Nesse enfoque, Montenegro ${ }^{2}$ afirma que excelência operacional é um caminho de melhoria contínua, um meio de aprimorar as competências essenciais da organização requer um esforço ordenado para aperfeiçoamento de padrões e práticas, envolvendo todos os processos da empresa, do planejamento de produção à entrega do produto ou serviço.

Segundo Montenegro ${ }^{2}$, a gestão de mudança no ambiente operacional envolve a seleção e implementação de um sistema integrado de iniciativas envolvendo todas as áreas de 
interface, levando a necessidade de preparação da equipe quanto aos aspectos comportamentais relacionados à mudança.

Em verdade, as mudanças realizadas em uma organização devem ter foco no aumento da capacidade produtiva dos processos. Nessa abordagem, Montenegro ${ }^{2}$ enuncia que maximizar a capacidade de produção é tema chave para a excelência operacional. Para tanto, um indicador em particular é extremamente útil, tratando da efetividade global do equipamento, ou simplesmente OEE - Overall Equipment Effectiveness.

Para melhor entendimento, Montenegro ${ }^{2}$ explica que OEE é um indicador do desempenho global de um ativo, mas é também uma medida de capacidade. Pode ser aplicado a um equipamento individual ou a uma planta industrial, e se compõe de três outros indicadores, que medem o rendimento, a produtividade e a qualidade.

Moreira $^{6}$ complementa as ideias apresentadas por Montenegro ${ }^{2}$, ao informar que o significado de OEE é o produto de três categorias: a disponibilidade, o desempenho e a qualidade. A disponibilidade é a medição das perdas por parada, o desempenho é medição das perdas por variação de ritmo e a qualidade é a medição das perdas por defeitos nos produtos.

Montenegro ${ }^{2}$ afirma que o objetivo deve ser, então, minimizar as perdas operacionais. Esse desafio é tanto maior, quanto maior for a complexidade do processo. Para atingi-lo é necessário, em primeiro lugar, garantir que o controle do processo seja feito sobre uma base de dados sólida, que garanta a integridade e confiabilidade das informações.

A redução das perdas operacionais aumenta o tempo de utilização de máquinas e equipamentos em uma empresa. Riztman et al. ${ }^{7}$ abordam em seus estudos que a utilização representa o tempo de operação total, ou seja, as horas trabalhadas, em relação ao tempo em que o equipamento estava disponível para operar. A utilização é função direta do planejamento e da logística das operações, bem como de fatores humanos.

Desta forma, a produtividade deve ser medida para controlar as perdas. Sobre produtividade, Stevesson ${ }^{8}$ relata que é uma medida da quantidade produzida durante certo intervalo de tempo de operação das horas trabalhadas. Afirma ainda que esta é, basicamente, consequência da eficiência operacional, da qualidade da matéria-prima processada e de perdas no processo. Quando se trata de equipamentos móveis em operações de extração de minério de ferro em mina a céu aberto, a produtividade é altamente afetada pelas condições de infraestrutura (condições de estradas e praças, de carga e descarga, planejamento de acessos), pelas características do material manuseado e até mesmo pela altitude e condições climáticas.

Neste contexto, esta pesquisa tem como objetivo verificar os resultados da aplicação de ações que almejam a excelência no processo de carga, descarga e transporte de minério de ferro de uma empresa situada na Região do Médio Piracicaba em Minas Gerais.

\section{MATERIAIS E MÉTODOS}

Esta pesquisa é um estudo de caso, realizada em uma empresa de mineração de minério de ferro a céu aberto, caracterizada como exploratória e documental.

O estudo foi realizado em uma empresa localizada na Região do Médio Piracicaba em Minas Gerais, no período entre agosto e outubro de 2016. A mineradora foi escolhida pelo método de acessibilidade, uma vez que um dos autores da pesquisa é funcionário da empresa. $\mathrm{O}$ estudo abrangeu a área de extração de minério de ferro, onde é realizado o carregamento, descarregamento e transporte do produto por meio de equipamentos e caminhões fora de estrada.

Para realização deste artigo, foram utilizados documentos e observação participativa de um dos autores da pesquisa. Assim, o processo foi observado e documentos da empresa foram analisados.

Um dos documentos refere-se às atas de reuniões ocorridas no período de julho de 2014 a junho 2015. Reuniões que foram realizadas pelos gestores e supervisores do processo de carga, descarga e transporte de minério para identificar falhas e apresentar ações que almejassem a excelência no processo produtivo. As atas foram analisadas e os dados foram 
coletados. Esses possuem natureza qualitativa e foram tratados pelo método de análise de conteúdo.

Dados de indicadores de utilização de equipamentos, taxa de produtividade e o volume de produção em toneladas foram retirados do relatório da empresa do período de julho de 2014 a junho 2016. Esses possuem natureza quantitativa.

\section{RESULTADOS E DISCUSSÃO}

A partir de julho de 2014, gestores e supervisores da área de carga, descarga e transporte do processo de extração de minério de ferro atentaram para realizar melhorias no processo. Isso para aumentar a produtividade e obter excelência na operação. Desta forma, reuniões foram realizadas para discutirem e identificarem as causas que impactavam as operações e, consequentemente, perdas no processo.

Com o início dos trabalhos, gestores e supervisores passaram a observar o processo com o propósito de identificar os desvios. Isso gerou o levantamento de várias falhas, que foram obtidas por meio brainstorming, realizada com a equipe de gestores e supervisores nas reuniões. As falhas identificadas foram:

- os operadores não são altamente qualificados;

- os parâmetros e limites operacionais dos equipamentos não são rigorosamente observados;

- os operadores não identificam desvios e não tomam as ações necessárias utilizando efetivamente os sistemas de monitoramento;

- não são utilizados recursos avançados para treinamento reciclagem e avaliação dos operadores;

- a qualidade da operação não é constantemente avaliada e as deficiências não são identificadas e eliminadas por meio de treinamentos específicos;

- não operam de forma a preservar a integridade do equipamento, observando sempre as condições operacionais;

- os operadores não realizam testes e reportam eventuais problemas;

- os procedimentos operacionais não são padronizados e não é de conhecimento geral;

- não existem atitudes proativas no sentido de garantir que os operadores estejam sempre alertas e em boas condições físicas e mentais para desempenhar suas atividades com segurança.

A partir dessas informações, foram desenvolvidas e implementadas ações para realizar melhorias e aumentar a produtividade no processo estudado. É de se evidenciar que a partir da identificação das não conformidades, ações foram definidas e desenvolvidas imediatamente no processo para sanar os desvios.

\subsection{Melhorias para aumentar a produtividade}

Neste tópico, foram descritas as ações implementadas para melhorar o desempenho do processo, voltadas ao desenvolvimento das equipes, incentivo à participação dos empregados, mudança comportamental e gerenciamento dos objetivos.

\subsubsection{Melhorias para os funcionários que atuam no processo de carga, descarga e transporte de minério de ferro}

Para aumentar o comprometimento da equipe de carga, descarga e transporte, aumentando o desenvolvimento, foi proposto e realizadas as seguintes ações:

- Envolver e motivar os empregados, objetivando um alto nível de compromisso;

- Definir equipes de trabalho multifuncionais envolvendo operação e manutenção, além de encorajar a participação; 
- Priorizar a educação e o treinamento, tanto no campo comportamental quando no técnico;

- Estabelecer metas comuns às turmas envolvidas na produção;

- Desdobrar e manter claramente as metas específicas de cada equipe;

- Manter controle efetivo quantos aos objetivos e metas;

- Promover a delegação de atividades para que os empregados tenham maior comprometimento e iniciativa para solução dos problemas;

- Desenvolver um forte senso de "propriedade" dos equipamentos: o operador deve comporta-se como "dono do equipamento;

- Desenvolver e manter um ambiente altamente participativo;

- Comunicar os resultados a todos os envolvidos;

- Reconhecer os bons operadores, incentivar o elogio e aprovar as iniciativas.

\subsubsection{Melhorias na gestão do desempenho dos equipamentos móveis}

Em relação ao desempenho dos equipamentos móveis, as ações propostas e desenvolvidas para realizar melhorias no processo foram:

- Selecionar o tipo correto do equipamento;

- Garantir o match (porte) adequado entre os equipamentos de carga e transporte. As Figuras 1 e 2 apresentam a carregadeira adequada ao tamanho do caminhão sendo carregado para o transporte.

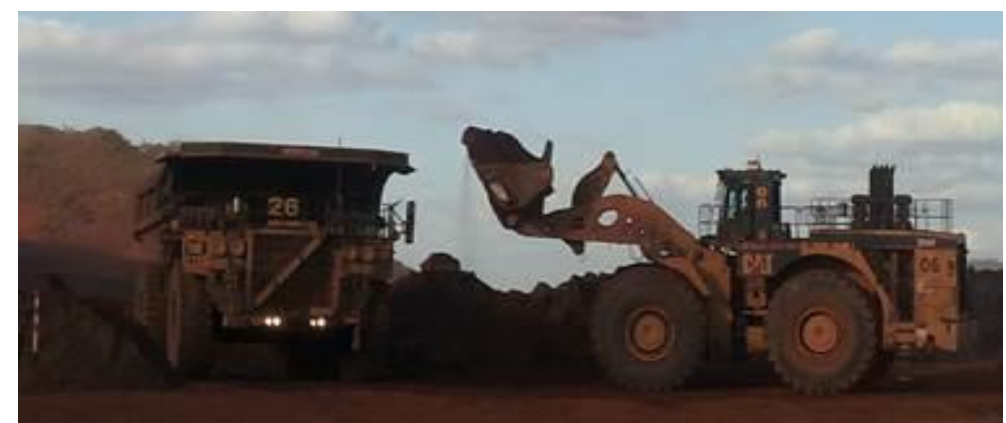

Figura 1 - Operação dos Equipamentos de Grande Porte. (Pesquisa Direta, 2017)

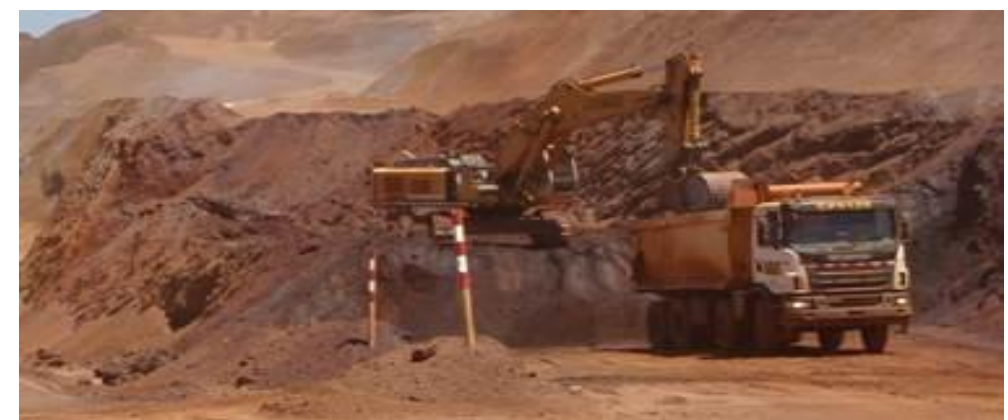

Figura 2 - Operação dos Equipamentos de Pequeno Porte. (Pesquisa Direta, 2017)

- Equipar corretamente as caçambas das carregadeiras para o tipo de caminhão;

- Avaliar as operações quanto à severidade de aplicação e desenvolver uma estratégia de operação e manutenção compatível;

- Configurar corretamente a área de trabalho ( carregamento e descarga). A Figura 3 mostra a preparação da área de carregamento; 


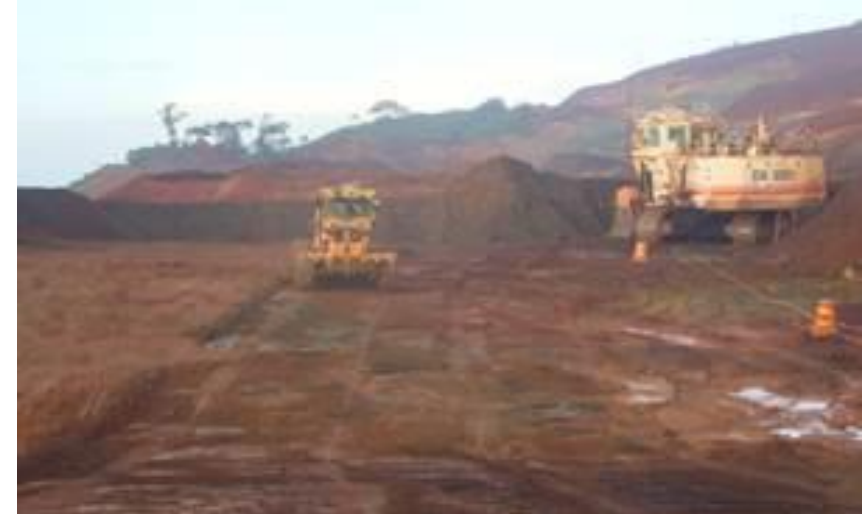

Figura 3 - Acerto da praça de carregamento por motoniveladora. (Pesquisa Direta, 2017)

- Garantir um bom planejamento e conservação de estradas e acesso. Assim, os acessos foram adequados, conforme mostra a Figura 4;

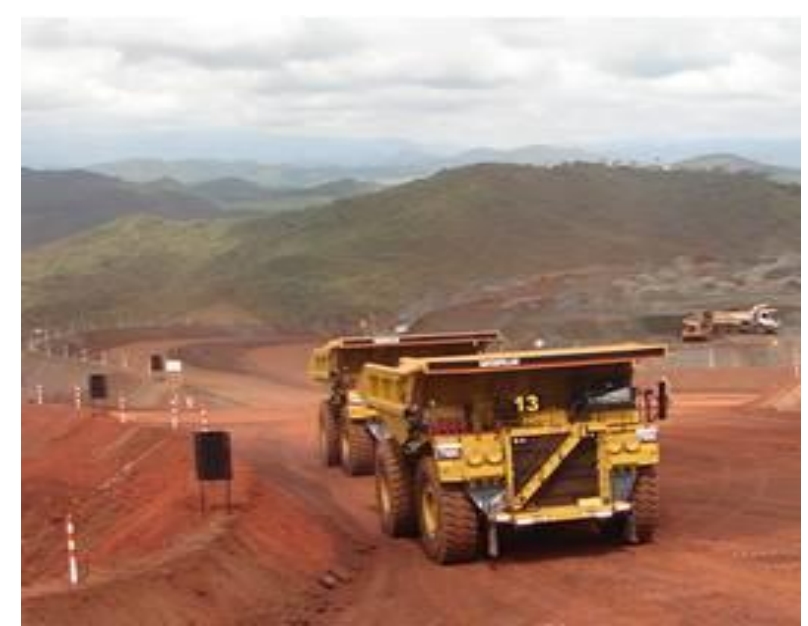

Figura 4 - Acesso em bom estado de conservação. (Pesquisa Direta, 2017)

- Aperfeiçoar o sistema de carregamento e transporte:

- Garantir que, a todo o momento, o número de caminhões e de máquinas de carga estejam balanceados;

- Definir a prioridade. Com um pequeno excesso de caminhões em relação às máquinas de carga, a efetividade do carregamento é maximizada. Por outro lado, o excesso de equipamentos de carga privilegia o transporte. Em qualquer situação, o objetivo é manter ambos - máquinas de carga e caminhões - operando com alto índice de produtividade;

- Implementar um sistema de despacho (roteirização) que trabalhe efetivamente na otimização dos parâmetros de fila e tráfego. Desta forma, o sistema de despacho, que realiza a roteirização dos equipamentos, foi implantado. Para isso, ocorreu mudança sistemática do posicionamento das antenas conforme praça de carga, descarga e via de tráfego;

- Selecionar o método de carregamento mais produtivo: foram avaliados os métodos de carregamento, pois sua influência sobre a produtividade é acentuada. No sistema tradicional, o caminhão é carregado sempre pelo mesmo lado. No sistema otimizado, o carregamento é feito pelos dois lados. Enquanto a carregadeira finaliza o carregamento de um lado, outro caminhão se posiciona do outro lado da máquina. Nesse caso, o tempo de manobra e de filas é reduzido e a operação de carregamento torna-se praticamente continua; 
- Gerenciar a velocidade dos caminhões - global e por trecho - identificando e corrigindo, rapidamente, eventuais restrições de velocidade;

- Minimizar os tempos de manobra, filas e outros atrasos operacionais;

- Gerenciar as cargas dos caminhões, procurando reduzir o desvio padrão das cargas e minimizando a presença de "mortos" na báscula. A Figura 5 mostra uma carregadeira realizando a limpeza da báscula do caminhão, isto é, retirando resto de material, que é denominado de "morto";

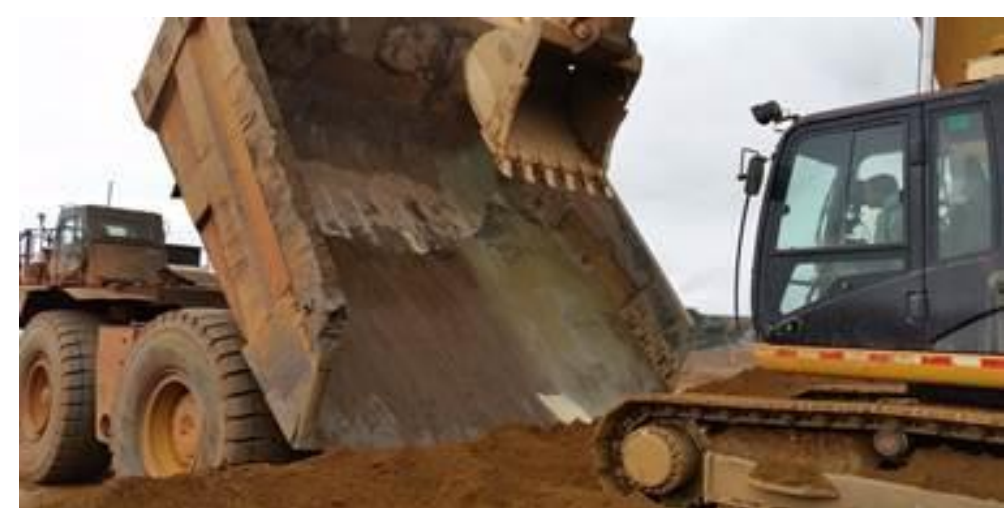

Figura 5 - Limpeza de báscula do caminhão, retirada de "morto". (Pesquisa Direta, 2017)

- Maximizar a utilização dos equipamentos;

- Realizar as trocas de turno rapidamente;

- Garantir uma atuação integrada do sistema de despacho junto à operação, manutenção e planejamento, a fim de minimizar as paradas dos equipamentos.

\subsection{Impacto das Melhorias nos Resultados de Desempenho Operacional}

Neste tópico, são analisados os resultados das ações implementadas para aumentar os indicadores de desempenho operacional.

Foi realizada uma análise dos indicadores de desempenho da frota de transporte composta de caminhões fora de estrada no período de julho de 2014 a junho de 2016. A Figura 6 apresenta os valores do índice de utilização física dos equipamentos de carga, descarga e transporte. Importante informar que os valores referem-se a um período de um ano, contados de julho a junho dos anos de 2014 a 2016.

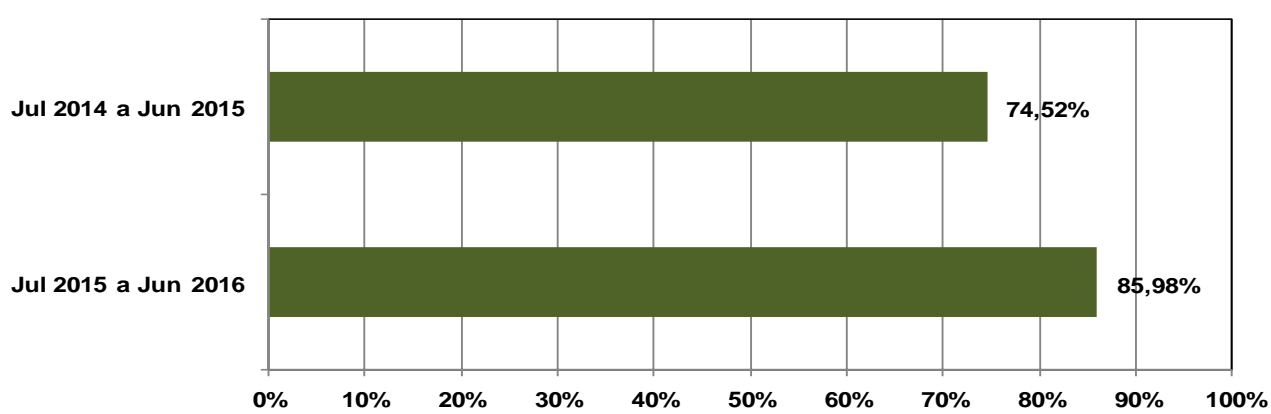

Figura 6 - Evolução anual do índice da Utilização Física em (\%)

Analisando o gráfico da Figura 6 pode-se notar que o percentual da utilização física dos caminhões fora de estrada, subiu significativamente após a implementação das ações para sanar os problemas identificados no processo. Obteve-se um aumento de $11,46 \%$ de julho de 2014 para junho de 2016.

A taxa de produtividade foi outro indicador de desempenho levantado neste estudo. Os valores de junho de 2014 a junho 2016 podem ser verificados pela Figura 7. 


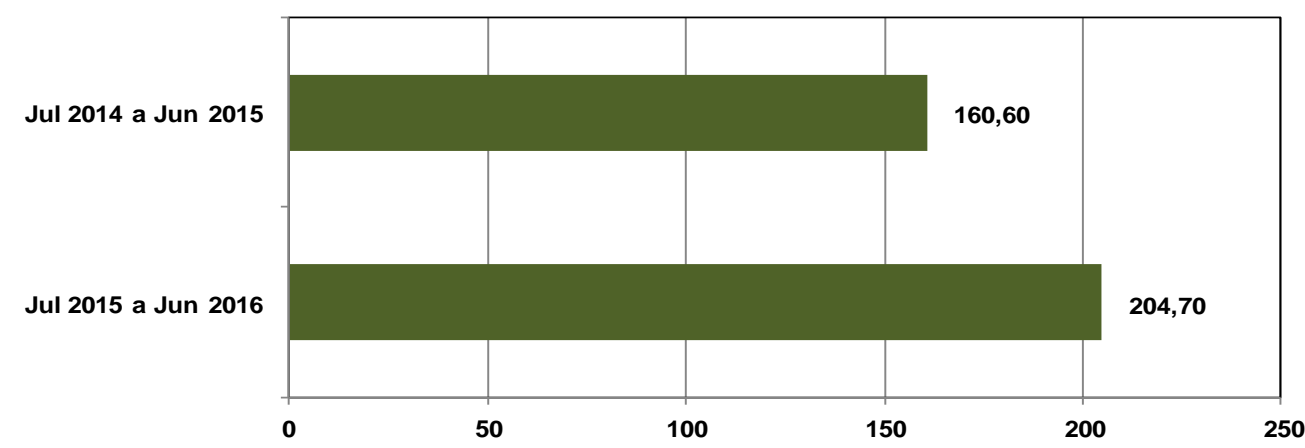

Figura 7 - Evolução Anual da taxa de Produtividade Horária em T/H.

Analisando a Figura 7 pode-se observar o aumento da produtividade horária, com um ganho de $27,5 \%$ no período de julho de 2014 para junho de 2016. O aumento se deve pela eliminação de diversos atrasos operacionais, tais como: fila na carga, locomoção não produzindo e aguardando equipamento de carga. Os atrasos operacionais impactam diretamente na produtividade horária. Com a implementação dos conceitos e métodos da excelência, foi possível garantir a efetividade na produtividade dos equipamentos, ou seja, a todo tempo que estão sendo utilizados, os caminhões estão produzindo.

Em sequência, a evolução anual da movimentação, ou seja, a quantidade de material transportado em toneladas, teve um melhor rendimento, conforme pode ser verificado pela Figura 8.

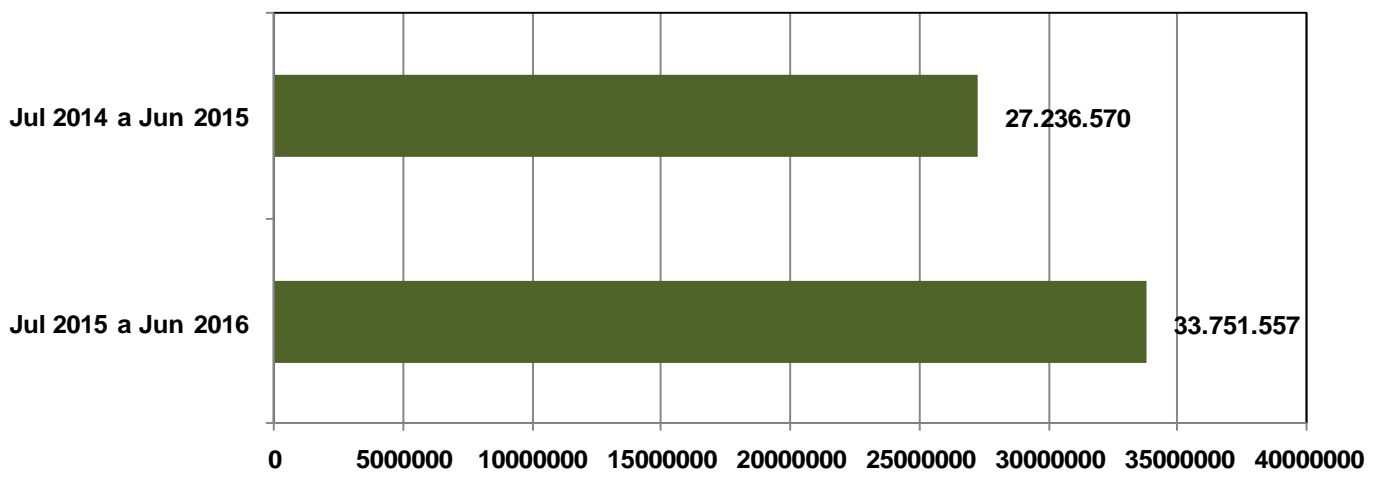

Figura 8 - Evolução anual da Movimentação (Produção) em toneladas.

Como consequência da evolução da utilização física e da produtividade horária, a movimentação total que é a produção realizada pelos caminhões, aumentou $23,92 \%$, o que garante que todos os caminhões estão com sua capacidade de produção maximizada, em decorrência da otimização de todos os indicadores de desempenho operacional. Com isso, pode-se verificar os ganhos com a aplicação das ações implementadas.

\section{CONCLUSÃO}

Com o desenvolvimento deste estudo de caso verificou-se que é papel da gestão das operações compatibilizar a demanda de recursos com os objetivos estratégicos da organização, em especial a necessidade de alcançar a maior eficiência no uso dos recursos: humanos, equipamentos, materiais, tecnologia e informações; visando a interação nos processos produtivos.

Desta forma, o aumento do desempenho dos equipamentos móveis foi analisado e definido que o domínio da operação destes equipamentos é fator preponderante para o alcance da efetividade operacional. A eficiência do operador é crucial para alcançar uma operação 
eficaz. A qualidade do esforço individual se transforma em contribuição coletiva, para que as metas ligadas aos indicadores de desempenho sejam alcançadas.

Pode-se afirmar, a partir das ações implementadas, que a excelência nas operações da utilização física dos equipamentos de carga, descarga e transporte foi alcançada. Importante enfatizar que ocorreu aumento $11,46 \%$ no período de dois anos. Esse resultado foi alcançado, devido à diminuição das horas improdutivas internas e externa, além da eliminação de várias falhas na operação.

Nota-se também, o aumento da produtividade horária, com um ganho de $27,5 \%$ no período de dois anos. Esse aumento aconteceu devido à eliminação de diversos atrasos operacionais, tais como: fila na carga, locomoção não produzindo e aguardando equipamento de carga. Os atrasos operacionais impactam diretamente na produtividade horária. Com a implementação dos conceitos e métodos de excelência, foi possível garantir a efetividade na produtividade dos equipamentos, ou seja, todo tempo que os caminhões estão sendo utilizados, estão produzindo.

Como consequência, da evolução dos indicadores de desempenho da utilização física e da produtividade horária, a movimentação total da produção realizada pelos caminhões fora de estrada, aumentou $23,92 \%$, o que garante que todos os caminhões estão com sua capacidade de produção maximizada. Com isso, pode-se verificar claramente, todos os ganhos de aplicação da excelência nas operações para melhoria contínua dos indicadores de desempenho operacional.

\section{REFERÊNCIAS}

1 FRANCIA, L. China continuará a derrubar o preço do minério de fero: política chinesa causou redução do consumo global e retraiu a demanda pelo insumo siderúrgico. Diário do Comércio. 2016. Disponível em: http://www.diariodocomercio.net.br/noticia.php?tit=china continuara a derrubar o pre co do minerio de ferro\&id=164789. Acesso em: 30/09/2016.

2 MONTENEGRO, I. Excelência Operacional: O desafio da melhoria contínua. São Paulo: Sobratema, 2007.

3 FUNDAÇÃO NACIONAL DA QUALIDADE. Conceitos Fundamentais da Excelência em Gestão. São Paulo: FNQ, 2006.

4 CORRÊA, C. A. \& CORRÊA, H. L. Administração de produção e operações, manufatura e serviços: uma abordagem estratégica. São Paulo: Atlas, 2006.

5 JURAN, Joseph M. A qualidade desde o projeto - novos passos para o planejamento da qualidade de produtos e serviços. São Paulo: Pioneira, 1992.

6 MOREIRA, D. Administração de produção e operações. São Paulo: Cengage Learnign, 2009.

7 KRAJEWSKI, L. J.; RITZMAN, L. Administração da produção e operações. São Paulo: Pearson Prentice Hall, 2008.

8 STEVENSON, W. J. Administração das operações de produção. Rio de Janeiro: LTC, 2001. 